\title{
Transcription factor expression as a predictor of colon cancer prognosis: a machine learning practice
}

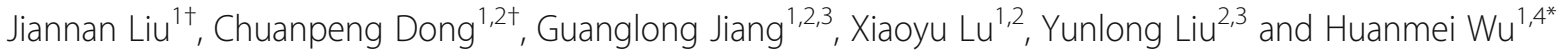

From The International Conference on Intelligent Biology and Medicine (ICIBM) 2019

Columbus, OH, USA. 9-11 June 2019

\begin{abstract}
Background: Colon cancer is one of the leading causes of cancer deaths in the USA and around the world. Molecular level characters, such as gene expression levels and mutations, may provide profound information for precision treatment apart from pathological indicators. Transcription factors function as critical regulators in all aspects of cell life, but transcription factors-based biomarkers for colon cancer prognosis were still rare and necessary.

Methods: We implemented an innovative process to select the transcription factors variables and evaluate the prognostic prediction power by combining the Cox PH model with the random forest algorithm. We picked five top-ranked transcription factors and built a prediction model by using Cox PH regression. Using Kaplan-Meier analysis, we validated our predictive model on four independent publicly available datasets (GSE39582, GSE17536, GSE37892, and GSE17537) from the GEO database, consisting of 925 colon cancer patients.

Results: A five-transcription-factors based predictive model for colon cancer prognosis has been developed by using TCGA colon cancer patient data. Five transcription factors identified for the predictive model is HOXC9, ZNF556, HEYL, HOXC4 and HOXC6. The prediction power of the model is validated with four GEO datasets consisting of 1584 patient samples. Kaplan-Meier curve and log-rank tests were conducted on both training and validation datasets, the difference of overall survival time between predicted low and high-risk groups can be clearly observed. Gene set enrichment analysis was performed to further investigate the difference between low and high-risk groups in the gene pathway level. The biological meaning was interpreted. Overall, our results prove our prediction model has a strong prediction power on colon cancer prognosis.

(Continued on next page)
\end{abstract}

\footnotetext{
* Correspondence: hw9@iupui.edu

† Jiannan Liu and Chuanpeng Dong contributed equally to this work. 'Depart of BioHealth Informatics, School of Informatics and Computing, Indiana University-Purdue University Indianapolis, Indianapolis, IN, USA

${ }^{4}$ Temple University College of Public Health, Philadelphia, PA, USA

Full list of author information is available at the end of the article
}

(c) The Author(s). 2020 Open Access This article is licensed under a Creative Commons Attribution 4.0 International License, which permits use, sharing, adaptation, distribution and reproduction in any medium or format, as long as you give appropriate credit to the original author(s) and the source, provide a link to the Creative Commons licence, and indicate if changes were made. The images or other third party material in this article are included in the article's Creative Commons. licence, unless indicated otherwise in a credit line to the material. If material is not included in the article's Creative Commons licence and your intended use is not permitted by statutory regulation or exceeds the permitted use, you will need to obtain permission directly from the copyright holder. To view a copy of this licence, visit http://creativecommons.org/licenses/by/4.0/ The Creative Commons Public Domain Dedication waiver (http://creativecommons.org/publicdomain/zero/1.0/) applies to the data made available in this article, unless otherwise stated in a credit line to the data. 


\begin{abstract}
(Continued from previous page)
Conclusions: Transcription factors can be used to construct colon cancer prognostic signatures with strong prediction power. The variable selection process used in this study has the potential to be implemented in the prognostic signature discovery of other cancer types. Our five TF-based predictive model would help with understanding the hidden relationship between colon cancer patient survival and transcription factor activities. It will also provide more insights into the precision treatment of colon cancer patients from a genomic information perspective.
\end{abstract}

Keywords: Colon cancer, Transcription factor, Machine learning, Cancer prognosis

\section{Background}

Colon cancer is the sixth in men and the fifth in women the most common cause of cancer-related death globally [1]. In the United States, colon cancer is estimated to have 135,430 newly diagnosed cases and result in 50,260 deaths in 2017, accounting for 9\% of cancer deaths [1]. Colon cancer is a complex disease with many risk factors, such as genetics, lifestyles, and dietary habits. Among them, inherited gene mutation, which can pass through family members, is one critical factor to increase one's colon cancer risk. A common colon cancer feature is the intra-cancer heterogeneity, which makes patients distinctive from each other in clinical presentations and responses to treatment. Colon cancer treatments should be tailored based on the individual's risk factors and genetic factors.

The inherited colon cancers can be broadly classified into two categories: familial adenomatous polyposis (FAP) and hereditary nonpolyposis colorectal cancer [2]. Molecular features in the genomics level play an essential role in treatment decision making and will continue providing more insights for pathological classification and tailored treatment for colon cancer. Proper colon cancer classification will significantly improve the survival rate, but hinders considerably by limited available prognosis assays.

Among the genetic factors, transcription factors (TFs) play a vital role in most important cellular processes, such as cell development, response to inner and outer environment change, cell cycle controls, and carcinogenesis. TFs are proteins that control the transcription of fragment DNA to messenger RNA by binding to specific DNA regions [3]. Their functions are to regulate, turn on and off genes to make sure that genes expressed in the right cell at the right time and in the right amount throughout the life of the cell and the organism [4]. For example, the NF- $\mathrm{kB}$ comprises a family of five TFs that form distinct protein complexes, which bind to consensus DNA sequences at promoter regions of responsive genes regulating cellular processes. NF- $\mathrm{kB}$ signaling and its mediated transcription play a critical role in inflammation and colorectal cancer development [5]. STAT3 is reported constitutively activated in colon-cancer-initiating cells and play a significant role in colon cancer progression [6]. FOXM1 was another TF that had been reported to be a key regulator of cell cycle progression, inflammation, tumor initiation and invasion [7].

In the past two decades, many researchers have implemented machine learning (ML) methods in the discovery and validation of cancer prognosis, especially after the population of High Throughput Technologies (HTTs) [8]. Recently, Long Nguyen Phuoc, et al. [9] developed a novel prognosis signature in colorectal cancer (CRC) by implementing several ML methods on public available CRC omics data. Their results demonstrated that the random forest method outperformed other ML methods they tried. Some researchers focused on microRNAs to find cancer prognosis signatures. Fatemeh Vafaee, et al. [10] proposed a prognostic signature of colorectal cancer comprising 11 circulating microRNAs. They also tested several different ML methods including RF and AdaBoost in their study. Their performance of the proposed prognostic signature was confirmed by an independent public dataset. Similarly, Jian Xu, et al. [11] developed a 4-microRNA expression signature for colon cancer patients by using the data from The Cancer Genome Atlas (TCGA). Their study showed that this 4-microRNA signature might play an important role in cancer cell growth after anti-cancer drug treatment. In 2016, Guangru $\mathrm{Xu}$, et al. [12] discovered a 15-gene signature that could effectively predict the recurrence and prognosis of colon cancer using a Support Vector Machine (SVM) algorithm. Their study pointed out that some genes in this signature might be an indicator of new therapeutic targets. Although these previous studies implemented machine learning methods on the discovery of cancer prognosis signatures, the crucial role of TFs has not been sufficiently addressed in cancer prognosis signature development.

The goal of our study is to identify the fundamental transcript factors, which are associated with clinical outcomes of colon cancer patients, by implementing an innovative cancer prognosis signature discovery process that combines the random forest algorithm with classic Cox Proportional Hazard (Cox PH) method. Our study will emphasize on only using TFs expression data to 
conduct prognostic analysis and we will provide a new perspective on how we can better use gene expression profiles to conduct prognostic research. By using proposed workflow, a TFs based prediction model has been successfully developed for colon cancer prognosis. The prediction power of our model is validated on hundreds of colon cancer patient samples available in the GEO database [13]. Our TF-based colon cancer prognosis prediction model can be used for a better classification of colon cancer patients in survival. Successful findings of this study will shed lights on understanding the mechanisms of the underlying colon cancer development and metastasis.

\section{Methods}

\section{Data sources}

In this study, we are using the expression data of TFs from two public resources. One is TCGA colon cancer (COAD) dataset, which can be downloaded from UCSC Xena (http://xena.ucsc.edu) [14] for both the expression dataset and the clinical data of patients. There are 497 samples in the COAD dataset, including 456 primary cancer tissue samples and 41 adjacent normal tissue samples. The downloaded TCGA level 3 RNAseq data is in the $\log 2$ (counts + offset) format. The TCGA COAD dataset is used as the training set in this study to build the predictive model for the colon cancer prognosis. Only patients carrying a primary tumor with the overall survival times and events were included in the training dataset. Then we further filtered the dataset by excluding patients who have missing information in cancer stage and other clinical information including sex and age. Finally, 435 patients with primary cancer tissue information were remaining in the training TCGA dataset,

The second public expression data resource is the microarray data from GEO database, which will be used to validate our prediction model. We chose four Affymetrix Human Genome U133 Plus 2.0 Array microarray study as validation datasets. The accession numbers, sequencing platform information, and sample sizes of each GEO dataset used in this study were listed in Table 1.

Table 1 Summary of the general clinicopathologic characteristics of patients in both training and testing datasets

\begin{tabular}{|c|c|c|c|c|c|}
\hline \multirow[t]{2}{*}{ Characteristic } & TCGA $(N=435)$ & GSE39582 $(N=563)$ & GSE17536 $(N=177)$ & GSE37892 $(N=130)$ & GSE17537 $(N=55)$ \\
\hline & N (\%) & N (\%) & N (\%) & N (\%) & N (\%) \\
\hline \multicolumn{6}{|l|}{ Age (years) } \\
\hline Median & 66 & 68 & 66 & 68 & 62 \\
\hline Range & $31-90$ & $22-97$ & $26-92$ & $22-97$ & 23-94 \\
\hline$<65$ & $166(38.2)$ & $211(37.5)$ & $78(44.1)$ & $54(41.5)$ & $32(58.2)$ \\
\hline$\geq 65$ & $269(51.8)$ & $351(62.3)$ & $99(55.9)$ & $76(58.5)$ & $23(41.8)$ \\
\hline \multicolumn{6}{|l|}{ Sex } \\
\hline Male & $202(46.4)$ & $309(54.9)$ & $96(54.2)$ & $69(53.1)$ & $26(47.3)$ \\
\hline Female & $233(53.6)$ & $253(44.9)$ & $81(45.8)$ & $61(46.9)$ & $29(52.7)$ \\
\hline \multicolumn{6}{|l|}{ T Status ${ }^{\mathrm{a}}$} \\
\hline $\mathrm{T} 1-2$ & 86 (19.8) & $56(9.9)$ & $N A$ & $N A$ & $N A$ \\
\hline T3-4 & $345(79.3)$ & $483(85.8)$ & $N A$ & $N A$ & $N A$ \\
\hline \multicolumn{6}{|l|}{ N Status ${ }^{a}$} \\
\hline NO & $254(58.4)$ & $299(53.1)$ & $N A$ & $N A$ & $N A$ \\
\hline N1 & $100(23.0)$ & $133(23.6)$ & $N A$ & $N A$ & $N A$ \\
\hline N2 & 78 (17.9) & $98(17.4)$ & $N A$ & $N A$ & $N A$ \\
\hline \multicolumn{6}{|l|}{ M Status $^{\mathrm{a}}$} \\
\hline MO & $318(73.1)$ & $479(85.1)$ & $N A$ & $N A$ & $N A$ \\
\hline M1 & $60(13.8)$ & $61(10.8)$ & $N A$ & $N A$ & $N A$ \\
\hline$M X$ & 47 (10.8) & $2(0.4)$ & $N A$ & $N A$ & $N A$ \\
\hline \multicolumn{6}{|l|}{ Stage } \\
\hline I & 73 (16.8) & $32(5.7)$ & 24 (13.6) & & $4(7.3)$ \\
\hline ॥ & 167 (38.4) & $262(46.5)$ & 57 (32.2) & 73 (56.2) & $15(27.3)$ \\
\hline III & $124(28.5)$ & $204(36.2)$ & 57 (32.2) & $57(43.8)$ & $19(34.5)$ \\
\hline IV & 60 (13.8) & 60 (10.7) & $39(22)$ & & 17 (30.9) \\
\hline
\end{tabular}

${ }^{\mathrm{a}} T$ status Describes the size of primary tissue and whether it has invaded nearby tissue, $N$ status Describes nearby lymph nodes that are involved, $M$ status Describes distant metastasis 
The respective clinical data were retrieved from published literature. The GEO dataset also filtered similarly to the TCGA COAD dataset with the survival events and times. In the end, the total number of GEO samples we used for prediction model validation is 1584. Before performing further analysis, the Affymetrix microarray data were normalized using the Robust Multi-array Average (RMA).

As shown in Table 1 for the summary of the training and testing datasets, there are substantial similarities upon patient diagnosed age, gender and in the AJCC staging level. The consistency in the pathology levels renders convincing for further analysis without bias or overfitting.

\section{Workflow of the study}

The overall workflow of our study is demonstrated in Fig. 1, which can be classified into three stages: TFs Screening, Predictive Modeling, and Model Validation. In Stage 1, we first identified a complete list of human TFs with official annotation from previous publications. Since not all the human TFs have the expression data in TCGA COAD dataset, the overlapped genes between TCGA COAD dataset and the complete list of TFs identified. Among the overlapping TFs, we further narrow down the numbers of TFs by the Cox PH Model analysis, which resulted in a limited set of TFs. Cox $\mathrm{PH}$ model is a widely used and performance proved statistical model in prognostic signature construction [8].

In Stage 2, since there are still too many colon prognosis TFs (more than $20 \mathrm{TFs}$ ), we need to decrease the final prognosis TFs to build a valid and good performance prognosis signature. The ensemble learning method, random forest method, is performed to refine further and reduce the TFs. Based on the RF training results, the most significant TFs are selected based on the top feature importance of RF. With the final TF list, we trained a predictive model for colon cancer prognosis using Cox PH regression.

Stage 3 is the validation of the predictive model. First, the prediction power is tested by accuracy analysis. Furthermore, the predictive model is validated on colon cancer datasets, collected from GEO database, including 925 samples from 4 studies. The Gene Set Enrichment Analysis (GSEA) [15] was also conducted to obtain further insights into our prediction model in the pathway level.

\section{Details on the variable selection and survival analysis methods}

In Stage 1 of the variable selection, we used the univariate Cox $\mathrm{PH}$ model in the statistical environment $\mathrm{R}$

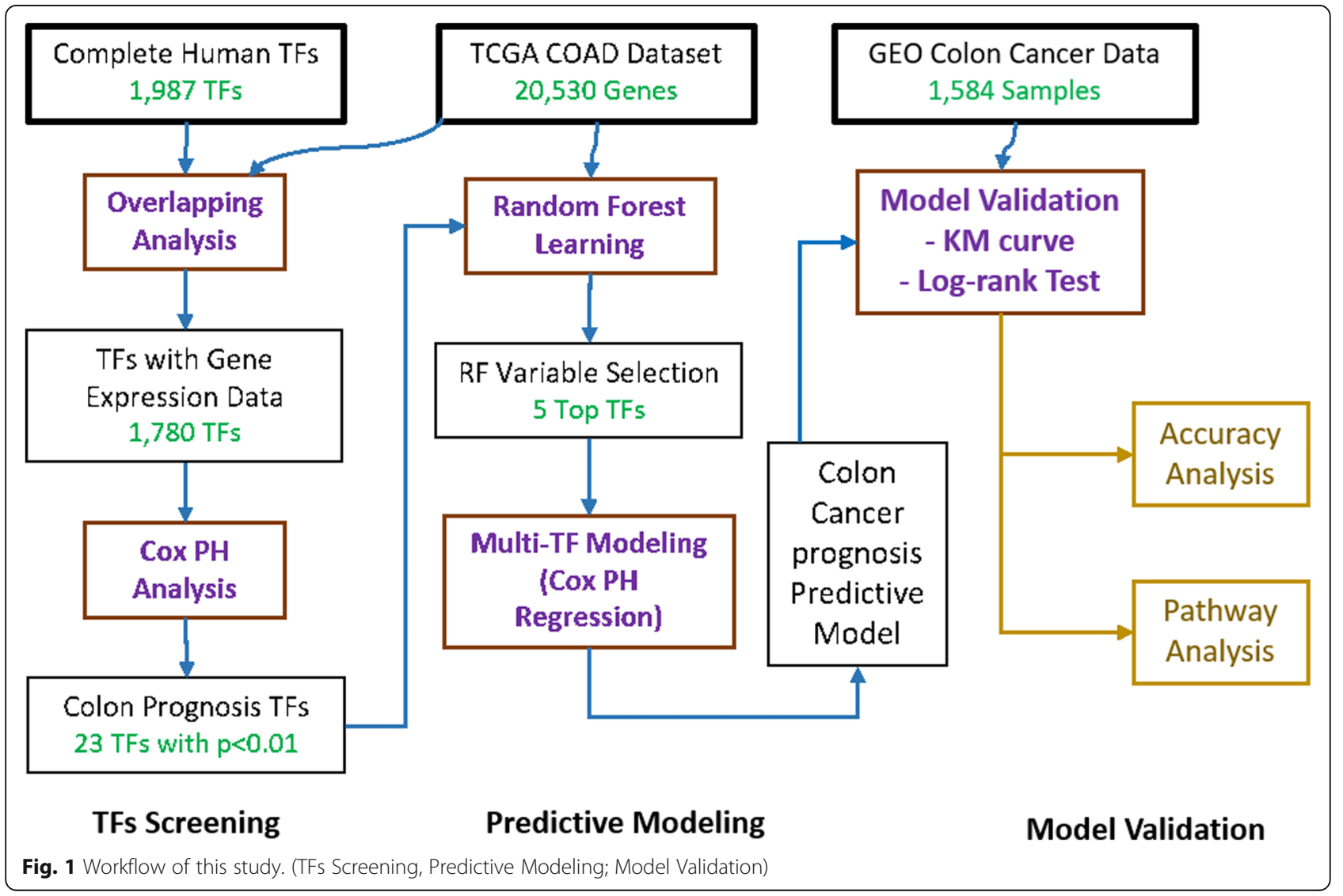


(v3.4), the association between expression profiles of TFs and the overall survival of patients was calculated to identify the prognostic ones. Any TF with a $p$-value less than 0.01 was considered statistically significant and used for further investigation.

In Stage 2 of refining variable selection, we performed RF methods for variable selection given that RF can be used for both classification problems and regression problems. RF [16] is an ensemble algorithm that use a bagging method to combine the multiple decision trees. It draws a set of samples from the whole dataset with replacement to feed the decision tree. After one decision tree has been trained, another sample set will be drawn from the whole dataset to train another decision tree. The process is repeated in the RF algorithm until the desired number of decision trees are trained. The final output of the prediction RF model can be the average of each decision tree' output. In cancer prognosis signature discovery practice, RF is a performance proved method $[9,10,17]$. In our study, the randomForestSRC for survival package [18] was used to measure the importance of each variable's contribution to the overall survival of colon cancer patients. This package uses minimal depth variable selection. The algorithm is the termed RSFVariable Hunting [19]. It exploits maximal subtrees for effective variable selection in survival data scenarios. In our implementation, the parameters used in the feature selection RF model were ntree $=1000$ and nstep $=5$.

In Stage 3, for the validation of the predictive model, the Kaplan-Meier (KM) curve [20] was used to estimate the difference in the survival between high and low risk groups in validation datasets. The log-rank test [21] was conducted to test the significance of the difference between subgroups since the log-rank test is a very robust statistical method to test important differences between two groups and is widely used in clinical trial experiments.

\section{Results}

\section{The results of identifying the potential prognostic} transcription factors

The complete list of 1987 human TFs was downloaded based on the census of human TFs from the Nature Review Genetics paper by Vaquerizas, Juan M., et al. [22]. Among the listed human TFs, 1834 of them have gene symbols annotations. After mapping to TCGA COAD dataset, only 1780 TFs have gene expression data in TCGA COAD dataset, which were included in this study.

The univariate Cox $\mathrm{PH}$ regression was applied to the gene expression profiles for the overlapping 1780 TFs and the patient clinical data in TCGA colon cohort, to identify the TFs, which are associated with the survival of the patients and have the potential using as prognostic markers. Those TFs with $p \leq 0.01$ were kept for further analysis (The selected 23 TFs are listed in Supplementary Table S1).

\section{Results on building the multi-TF predictive model}

To identify the minimum subset of TFs that can still achieve a good prediction of colon cancer survival, the 23 TFs from the Cox PH regression model were further evaluated with a random forest algorithm, randomForestSRC. In the randomForestSRC variable hunting mode, top $\mathrm{P}$ ranked variables will be selected, $\mathrm{P}$ is the average model size and variables are ranked by frequency of occurrence. In our study, five TFs (i.e., HOXC9, ZNF556, HEYL, HOXC4, and HOXC6) were chosen for the final predictive model construction. The results of the algorithm is shown in Fig. 2. The parameters for random forest are ntree $=1000$ and nstep $=5$.

To establish a multiple molecular based regression model, the multivariate Cox $\mathrm{PH}$ regression was trained with gene expression data using the five TFs and clinical variables from TCGA COAD dataset. The coefficients from the Cox model were then applied to a multivariate linear regression model. The risk score was calculated with the following formula:

$$
\begin{aligned}
\text { Risk score }= & 0.139 * \mathrm{HOXC6}-0.046 * \mathrm{HOXC} 4 \\
& +0.165 * \mathrm{HEYL} \\
& +0.106 * \mathrm{ZNF} 556-0.032 * \mathrm{HOXC} 9
\end{aligned}
$$

The final coefficients of the model have been modified automatically to achieve better performance and to increase accuracy overall. Thus, the coefficients of HOXC9 and $\mathrm{HOXC} 3$ are adjusted to slightly below zero, which are much smaller than those positive coefficients. Then we performed the KM analysis and the log-rank test result over these five selected TFs. The results and the $p$-value from previous Cox $\mathrm{PH}$ analysis, along with the hazard ratio for each of these genes are summarized in Fig. 3. It can be seen that all selected 5 TFs has Cox $p$-value $<0.01$, which indicates all these TFs are highly related to the overall survival of patients according to Cox $\mathrm{PH}$ analysis. For the log-rank p, only the ZNF556 has a $p$-value of 0.107 , while all the other four have $p$-value $<0.05$. According to the RF results, the importance of ZNF556 is ranked fourth in all 23 TFs with no significant difference with other TFs in maximum depth (Fig. 2), this qualifies the ZNF556 as one of the most important prognostic TFs. The Hazard ratios of all these five TFs are more than 1.0, indicating higher risks of colon cancer prognosis.

\section{Results on validation of the five-TF based prediction model}

Based on the median value of the predicted risks scores of all the patients in both the training and validation set, 


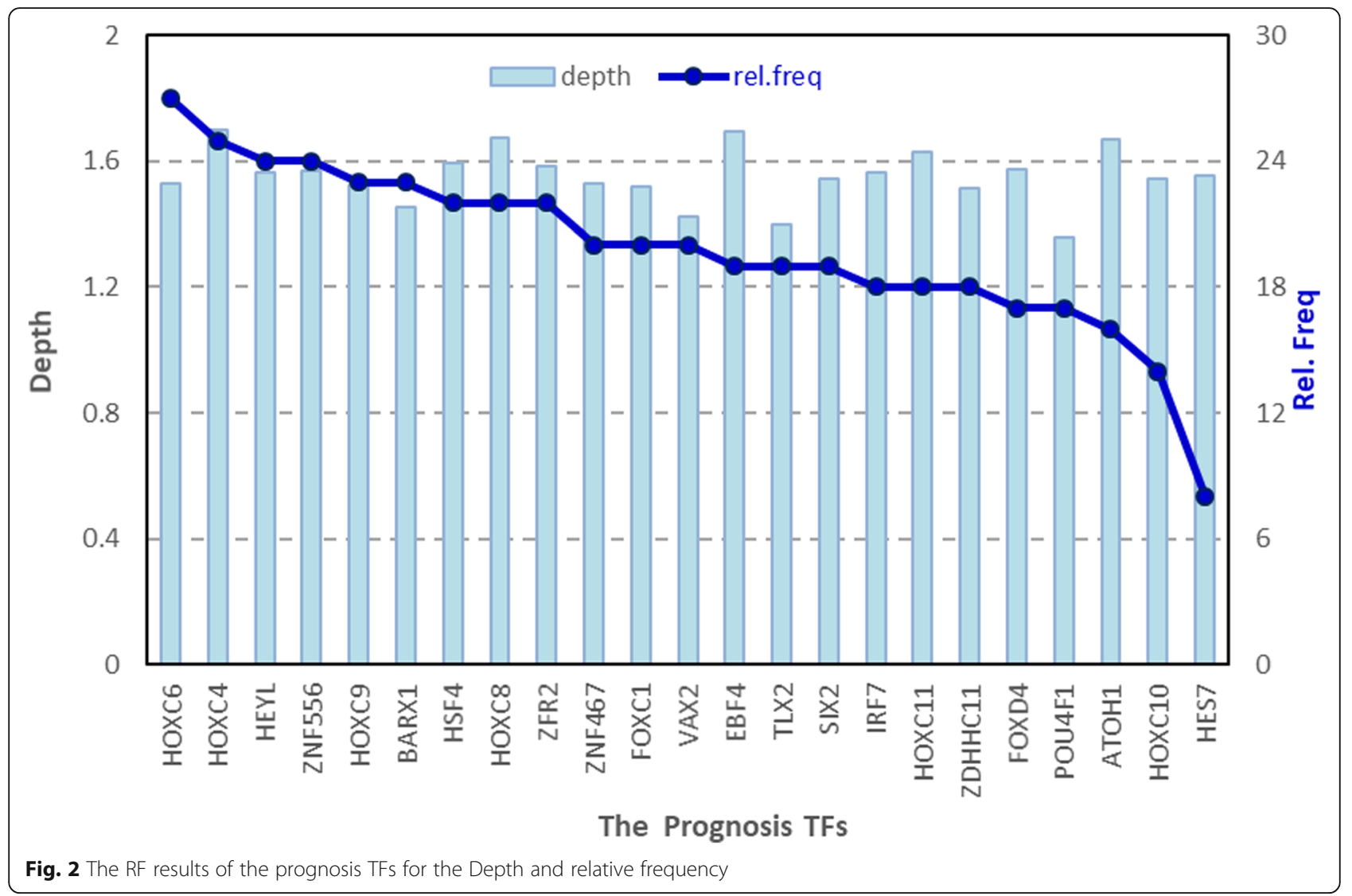

patients are classified into high-risk and low-risk subgroups. KM curve analysis and log-rank test were conducted to evaluate the performance of predicting power in colon cancer prognosis on TCGA COAD dataset. The results are shown in Fig. 4. The scatter plot (Fig. 4A(b)) shows the distribution of patients' overall survival status. The red point indicates the patient belonging to a high-risk group while a blue point indicates the patient belonging to a low-risk group. From the scatter plot, we can observe that the red points are more concentrated in the lower part of the figure. This is an indication that high-risk patients have a shorter survival time comparing to low-risk patients. The heatmap (Fig. $4 \mathrm{~A}(\mathrm{c})$ ) shows that the five selected TFs in our predictive model were highly expressed in TCGA COAD dataset. Moreover, the KM curve (Fig. 4B) shows a distinctive survival difference between the high-risk and low-risk groups in a time span of more than 10 years. All these results prove the prediction power of our predictive model on TCGA COAD dataset.

To test the five-TF based signature as colon cancer survival predictor, we further validated the predictive model on another four independent microarray datasets

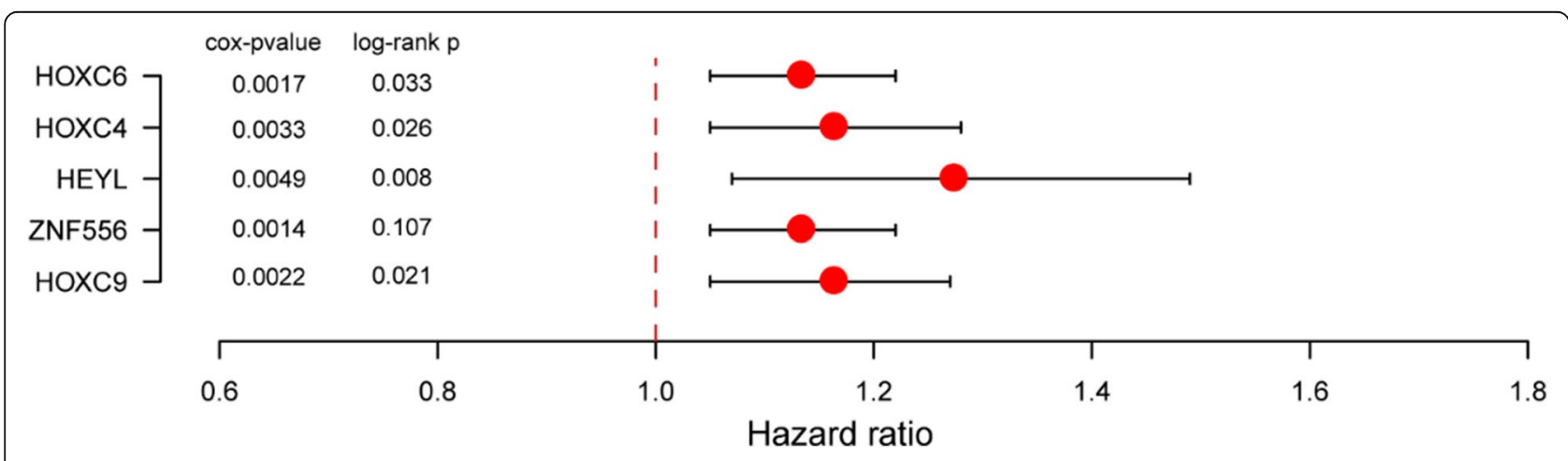

Fig. 3 Information on five prognostic TFs finally selected for building the prediction model 


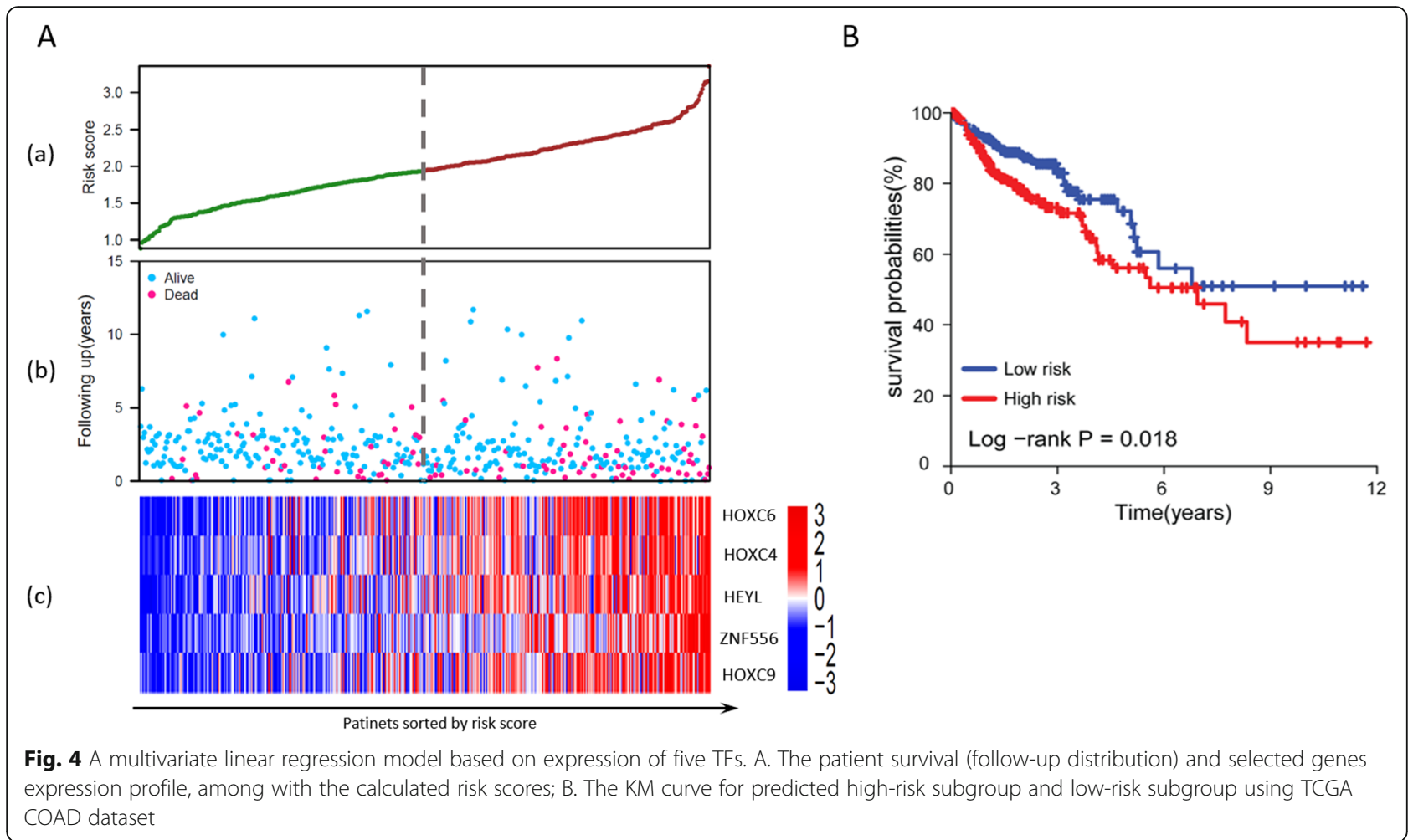

with a total of 1584 samples for GEO with GSE39582 $(n=563), \operatorname{GSE} 17536(n=177), \operatorname{GSE} 37892(n=130)$ and GSE17537 $(n=55)$. The risk score of each patient in validation dataset was calculated by using the same formula established with TCGA training dataset. The same coefficients were utilized to assign weight to each of the selected TF. By using the same median cutoff strategy to divide patients to the high-risk and low-risk groups, the KM curve analysis shows the consistent patterns with the TCGA COAD dataset. Patients in the high-risk group have a significantly shorter survival time than patients in the low-risk group (Fig. 5a-d), which suggests the clinical robustness among multiple centers. Therefore, our five-TF based signature is proved to be a robust predictor for colon cancer survival.

\section{Results on pathway analysis}

The Gene Set Enrichment Analysis (GSEA) [15] was conducted to investigate the biological function of this five-TF based signature, including its molecular function and gene-gene network. GSEA is performed on the TCGA COAD dataset with predicted high-risk subgroup versus low-risk subgroup. In conducting the GSEA study, the reference gene pathway database is the Kyoto Encyclopedia of Genes and Genomes (KEGG) pathway database [23]. The GSEA number of permutations is set to be 1000 , and the phenotype labels are determined according to whether a patient is in the high-risk subgroup or the low-risk subgroup. As illustrated in Fig. 6, the GSEA results showed that several cancer-related pathways were alternated in patients with high-risk scores, such as the pathways for the Epithelial-mesenchymal transition, the ECM receptor interaction, the cytokine-cytokine receptor interaction, and the cell adhesion molecules (Fig. 5ad). Taken together these findings, it's indicated that the five TFs in our model may highly associate with tissue morphogenesis, intercellular regulations and cell adhesion. By affecting these cell processes, these TFs may promote the tissue malignant then result in a poor overall survival rate of colon cancer patients.

\section{Discussions}

We implemented an innovative machine learning approach for signature variables, which combines the Cox $\mathrm{PH}$ method with the random forest algorithm. Our signature selection process can find the minimum subset of TFs to build the prognosis prediction model with satisfying performance. A five-TF predictive model was developed by training the classifiers on TCGA COAD dataset. The trained multivariable linear predictive model was validated with multiple datasets from the GEO database.

Three out of the five selected genes, i.e., HOXC4, HOXC6, and HOXC9, belong to the homeobox family of genes. The homeobox genes are highly conserved TF family and play an essential role in morphogenesis in all 


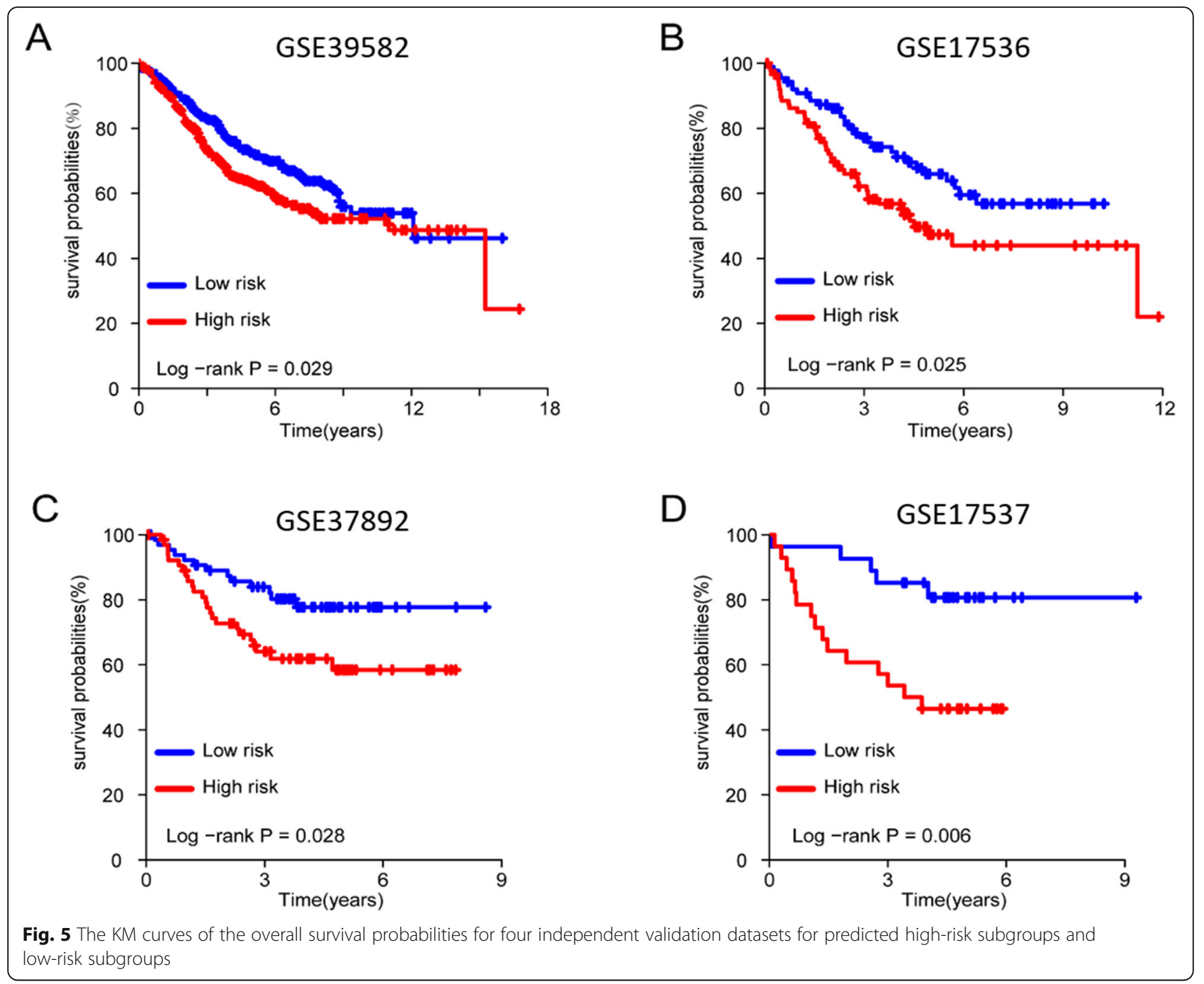

multicellular organisms. Dysregulation of HOX gene expression implicated as a factor in malignancies, and up-regulation has been observed in malignant prostate cell lines and lymph node metastases [24]. HOXC6 was also reported to be overexpressed in colorectal cancer tissue, and highly correlated with poor survival outcome and acts as a significant prognostic risk factor [25].

For the other two genes selected in our predictive model, HEYL belongs to the hairy and enhancer of split-related (HESR) family of basic helix-loop-helix (bHLH)-type transcription factor. A recent study shows that HEYL may be a tumor suppressor of liver carcinogenesis through upregulation of P53 gene expression and activation of P53-mediated apoptosis [26]. ZNF556 belongs to zinc finger protein (ZNF) family. Despite the large size of ZNF gene family, the number of disease-linked genes in this family is very small [27]. To the best of our knowledge, the research on ZNF556 related to cancer is very limited.
Therefore, our study provided new insight on potential relationships between overexpression of ZNF556 and the development of colon cancer.

Our study also showed that by using TFs to build a predictive signature for colon cancer prognosis is practical. The prediction power of the model is promising. Intuitively, the TFs have the overall control on the gene expressions in cells so that a TF-based predictive model should be able to indicate the different gene expression levels in some cancer types with high accuracy.

Our innovative signature discovery process can potentially be extended on other cancer types such as breast cancer or lung cancer. It will be interesting to carry out studies on whether these five TFs used by our model have tissue specific expression patterns in colon cancer. Moreover, by conducting downstream analysis such as gene regulation network analysis, we can probably identify genes that are regulated by our five TFs, these downstream genes can be potentially 


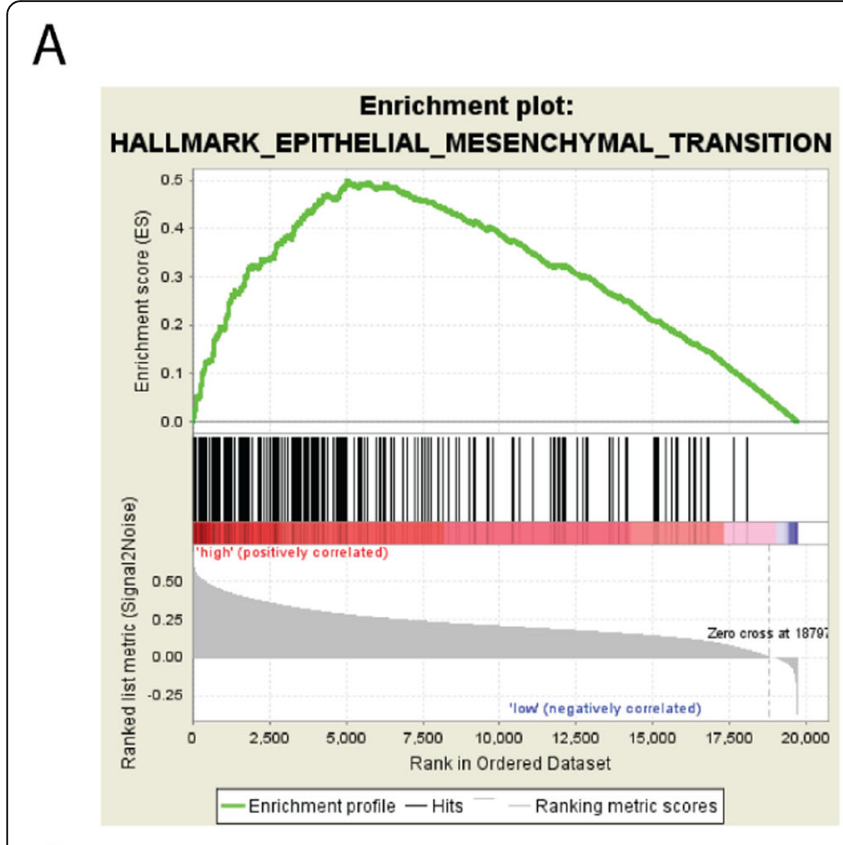

B

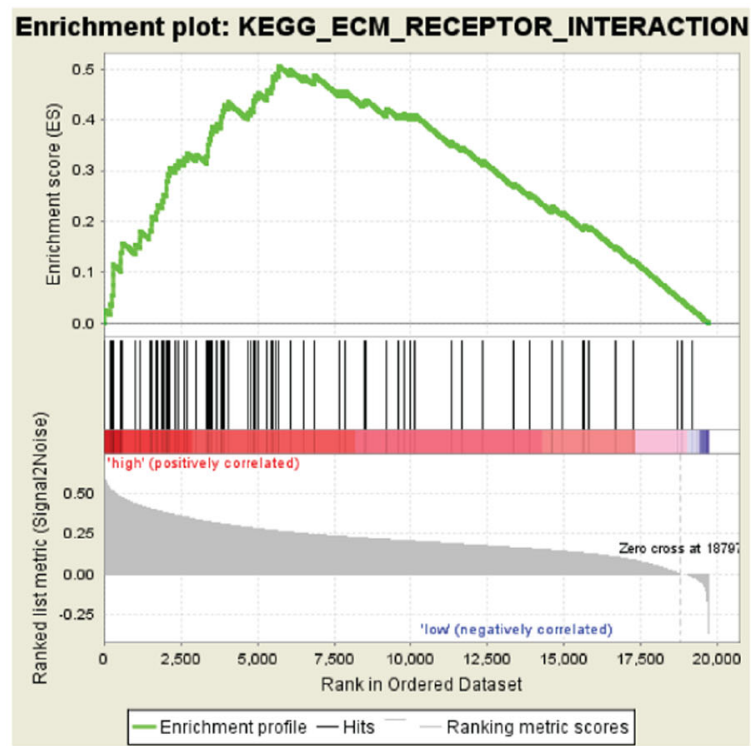

C

Enrichment plot:

D
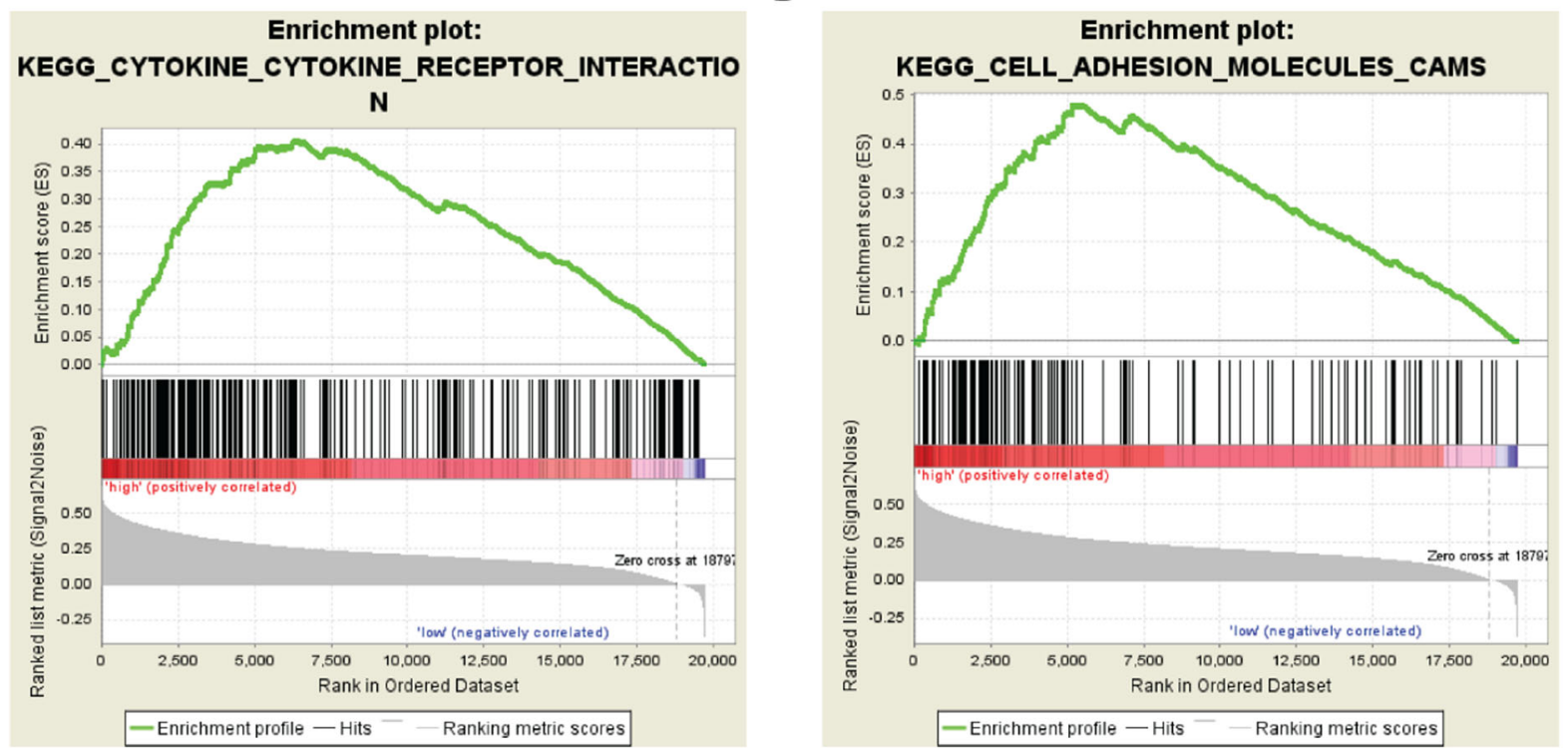

Fig. 6 Enrichment plots for the top four enriched gene pathways according to the GSEA results. GSEA is performed on TCGA COAD dataset

added to the prediction model to add more robustness to our model. Another future study is to examine the performance of combining traditional statistical methods, such as Cox $\mathrm{PH}$, with other machine learning methods, such as the artificial neural network (ANN) [28], to select potential prognostic TFs or other signatures for different types of cancer.

\section{Conclusion}

We have successfully identified a five-TF signature and built a predictive model for colon cancer prognosis signature with the selected five TFs by using a machine learning approach. Our five-TFs based linear model was validated on hundreds of publicly available patient data from the GEO database. The results showed that our model has a good predicting power in predicting colon cancer overall survival. Our predictive model and its biological functions would provide more insights in the precision treatment of colon cancer, which leads to further investigation on these five TF genes and their roles during the development of colon cancer at the molecular level. 


\section{Supplementary information}

Supplementary information accompanies this paper at https://doi.org/10. 1186/s12920-020-00775-0.

Additional file 1: Table S1. The 23 transcript factors identified as prognostic features.

\section{Abbreviations}

TF: Transcription factor; ML: Machine learning; RF: Random forest; TCGA: The cancer genome atlas; GEO: Gene expression omnibus; KEGG: Kyoto Encyclopedia of Genes and Genomes; Cox PH: Cox proportional hazard; KM: Kaplan-Meier; GSEA: Gene set enrichment analysis

\section{Acknowledgements}

Not applicable.

\section{About this supplement}

This article has been published as part of BMC Medical Genomics Volume 13 Supplement 9, 2020: The International Conference on Intelligent Biology and Medicine (ICIBM) 2019: Computational methods and application in medical genomics (part 2). The full contents of the supplement are available online at https://bmcmedgenomics.biomedcentral.com/articles/supplements/ volume-13-supplement-9.

\section{Authors' contributions}

$J L$ and CD contributed equally to this work. $J$ and CD designed the study, did the experiment and wrote the manuscript. GJ and XL processed the data and tested the code. HW and YL supervised the project and revised the manuscript. All authors read and approved the final manuscript.

\section{Funding}

Partially supported by the Research Contract from Indiana Primary Health Care Association (Wu). This article has not received sponsorship for publication.

\section{Availability of data and materials}

TCGA COAD dataset can be downloaded from http://xena.ucsc.edu, GEO datasets used in this study can be downloaded from GEO database by using access numbers: GSE39582, GSE17536, GSE37892, GSE17537.

\section{Ethics approval and consent to participate}

Not applicable.

\section{Consent for publication}

Not applicable.

\section{Competing interests}

The authors declare that they have no competing interests.

\section{Author details}

'Depart of BioHealth Informatics, School of Informatics and Computing, Indiana University-Purdue University Indianapolis, Indianapolis, IN, USA. ${ }^{2}$ Center for Computational Biology and Bioinformatics, Indiana University School of Medicine, Indianapolis, IN, USA. ${ }^{3}$ Department of Medical and Molecular Genetics, Indiana University School of Medicine, Indianapolis, IN, USA. ${ }^{4}$ Temple University College of Public Health, Philadelphia, PA, USA.

Published: 21 September 2020

\section{References}

1. Siegel RL, Miller KD, Jemal A. Cancer statistics, 2017. CA Cancer J Clin. 2017; 67(1):5

2. Ponz dLM, Sassatelli R, Benatti $P$, Roncucci L. Identification of hereditary nonpolyposis colorectal cancer in the general population. The 6-year experience of a population-based registry. Cancer. 1993;71(11):3493-501.

3. Lee Tl, Young RA. Transcription of eukaryotic protein-coding genes. Annu Rev Genet. 2000;34(1):77-137

4. Latchman DS. Transcription factors: an overview. Int J Exp Pathol. 1997;74(5): 1305-12.
5. Wang S, Liu Z, Wang L, Zhang X. NF-KB signaling pathway, inflammation and colorectal cancer. Cell Mol Immunol. 2009;6(5):327-34.

6. Lin L, Liu A, Peng Z, Lin HJ, Li PK, Li C, et al. STAT3 is necessary for proliferation and survival in colon cancer-initiating cells. Cancer Res. 2011;71(23):7226-37.

7. Wan LY, Deng J, Xiang XJ, Zhang L, Yu F, Chen J, et al. miR-320 enhances the sensitivity of human colon cancer cells to chemoradiotherapy in vitro by targeting FOXM1. Biochem Biophys Res Commun. 2015;457(2):125-32.

8. Kourou K, Exarchos TP, Exarchos KP, Karamouzis MV, Fotiadis DI. Machine learning applications in cancer prognosis and prediction. Comput Struct Biotechnol J. 2015;13:8-17.

9. Long NP, Park S, Anh NH, Nghi TD, Yoon SJ, Park JH, Lim J, Kwon SW. Highthroughput omics and statistical learning integration for the discovery and validation of novel diagnostic signatures in colorectal cancer. Int J Mol Sci. 2019;20(2):296

10. Vafaee F, Diakos C, Kirschner MB, Reid G, Michael MZ, Horvath LG, AlinejadRokny H, Cheng ZJ, Kuncic Z, Clarke S. A data-driven, knowledge-based approach to biomarker discovery: application to circulating microRNA markers of colorectal cancer prognosis. NPJ Syst Biol Appl. 2018;4(1):20.

11. Xu J, Zhao J, Zhang R. Four microRNAs signature for survival prognosis in colon cancer using TCGA data. Sci Rep. 2016;6:38306.

12. Xu G, Zhang M, Zhu H, Xu J. A 15-gene signature for prediction of colon cancer recurrence and prognosis based on SVM. Gene. 2017:604:33-40.

13. Barrett T, Wilhite SE, Ledoux P, Evangelista C, Kim IF, Tomashevsky M, Marshall KA, Phillippy KH, Sherman PM, Holko M, Yefanov A. NCBI GEO: archive for functional genomics data sets—update. Nucleic Acids Res. 2012;41(D1):D991-5.

14. Goldman M, Craft B, Swatloski T, Cline M, Morozova O, Diekhans $M_{\text {, et al }}$ The UCSC cancer genomics browser: update 2015. Nucleic Acids Res. 2015; 43(Database issue):D812-D7.

15. Subramanian A, Tamayo P, Mootha VK, Mukherjee S, Ebert BL, Gillette MA, Paulovich A, Pomeroy SL, Golub TR, Lander ES, Mesirov JP. Gene set enrichment analysis: a knowledge-based approach for interpreting genomewide expression profiles. Proc Natl Acad Sci. 2005;102(43):15545-50.

16. Liaw A, Wiener M. Classification and regression by randomForest. R News. 2002;2(3):18-22

17. Liu G, Dong C, Wang X, Hou G, Zheng Y, Xu H, Zhan X, Liu L. Regulatory activity based risk model identifies survival of stage II and III colorectal carcinoma. Oncotarget. 2017:8(58):98360.

18. Ishwaran $H$, Kogalur UB. randomForestSRC: random forests for survival, regression and classification (RF-SRC); 2016.

19. Ishwaran H, Kogalur UB, Gorodeski EZ, Minn AJ, Lauer MS. High-dimensional variable selection for survival data. Publ Am Stat Assoc. 2010;105(489):205-17.

20. Kaplan EL, Meier P. Nonparametric estimation from incomplete observations. J Am Stat Assoc. 1958;53(282):457-81.

21. Peto $R$, Peto J. Asymptotically efficient rank invariant test procedures. J R Stat Soc Ser A Gen. 1972;135(2):185-98.

22. Vaquerizas JM, Kummerfeld SK, Teichmann SA, Luscombe NM. A census of human transcription factors: function, expression and evolution. Nat Rev Genet. 2009;10(4):252.

23. Kanehisa M, Goto S. KEGG: Kyoto encyclopedia of genes and genomes. Nucleic Acids Res. 2000:28(1):27-30.

24. Miller GJ, Miller HL, van Bokhoven A, Lambert JR, Werahera PN, Schirripa O, Lucia MS, Nordeen SK. Aberrant HOXC expression accompanies the malignant phenotype in human prostate. Cancer Res. 2003;63(18):5879-88.

25. Ji M, Feng Q, He G, Yang L, Tang W, Lao X, et al. Silencing homeobox C6 inhibits colorectal cancer cell proliferation. Oncotarget. 2016;7(20):29216-27.

26. Kuo KK, Jian SF, Li YJ, Wan SW, Weng CC, Fang K, Wu DC, Cheng KH. Epigenetic inactivation of transforming growth factor- $\beta 1$ target gene HEYL, a novel tumor suppressor, is involved in the P53-induced apoptotic pathway in hepatocellular carcinoma. Hepatol Res. 2015:45(7):782-93.

27. Stevens SJ, van Essen AJ, van Ravenswaaij CM, Elias AF, Haven JA, Lelieveld SH, Pfundt R, Nillesen WM, Yntema HG, van Roozendaal K, Stegmann AP. Truncating de novo mutations in the Krüppel-type zinc-finger gene ZNF148 in patients with corpus callosum defects, developmental delay, short stature, and dysmorphisms. Genome Med. 2016;8(1):131.

28. Ching T, Zhu X, Garmire LX. Cox-nnet: an artificial neural network method for prognosis prediction of high-throughput omics data. PLoS Comput Biol. 2018;14(4):e1006076

\section{Publisher's Note}

Springer Nature remains neutral with regard to jurisdictional claims in published maps and institutional affiliations. 